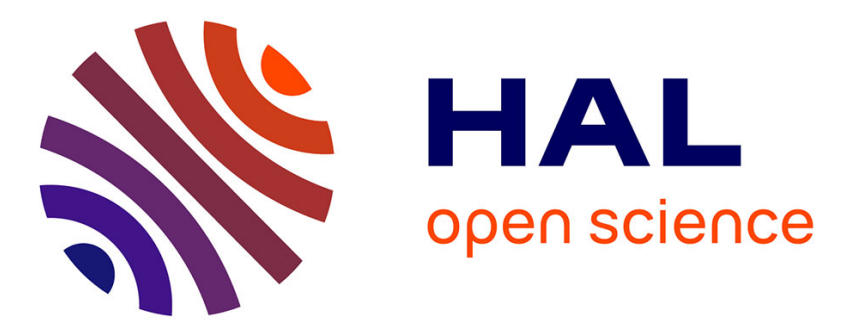

\title{
Approximations for All-to-all Uniform Traffic Grooming on Unidirectional Ring
}

Jean-Claude Bermond, David Coudert, Benjamin Lévêque

\section{To cite this version:}

Jean-Claude Bermond, David Coudert, Benjamin Lévêque. Approximations for All-to-all Uniform Traffic Grooming on Unidirectional Ring. Journal of Interconnection Networks, 2008, 9 (4), pp.471486. 10.1142/S0219265908002394 . inria-00429217

\section{HAL Id: inria-00429217 https://hal.inria.fr/inria-00429217}

Submitted on 1 Nov 2009

HAL is a multi-disciplinary open access archive for the deposit and dissemination of scientific research documents, whether they are published or not. The documents may come from teaching and research institutions in France or abroad, or from public or private research centers.
L'archive ouverte pluridisciplinaire HAL, est destinée au dépôt et à la diffusion de documents scientifiques de niveau recherche, publiés ou non, émanant des établissements d'enseignement et de recherche français ou étrangers, des laboratoires publics ou privés. 


\title{
Approximations for All-to-all Uniform Traffic Grooming on Unidirectional Ring*
}

\author{
Jean-Claude Bermond, David Coudert \\ Mascotte, I3S(CNRS/UNSA)-INRIA \\ Sophia Antipolis, France \\ \{firstname.lastname\}@sophia.inria.fr
}

\author{
Benjamin Lévêque \\ Laboratoire G-SCOP \\ Grenoble, France \\ benjamin. leveque@g-scop.inpg.fr
}

\begin{abstract}
Traffic grooming in a WDM network consists of assigning to each request (lightpath) a wavelength with the constraint that a given wavelength can carry at most $\mathrm{C}$ requests or equivalently a request uses $1 / C$ of the bandwidth. $C$ is known as the grooming ratio. A request (lightpath) needs two SONET add-drop multiplexers (ADMs) at each end node; using grooming, different requests can share the same $\mathrm{ADM}$. The so called traffic grooming problem consists of minimizing the total number of ADMs to be used (in order to reduce the overall cost of the network). Here we consider the traffic grooming problem in WDM unidirectional rings which has been recently shown to be APX-hard and for which no constant approximations are known. We furthermore suppose an all to all uniform unitary traffic. This problem has been optimally solved for specific values of the grooming ratio, namely $C=2,3,4,5,6$. In this paper we present various simple constructions for the grooming problem providing approximation of the total number of ADMs with a small constant ratio. For that we use the fact that the problem corresponds to a partition of the edges of the complete graph into subgraphs, where each subgraph has at most $C$ edges and where the total number of vertices has to be minimized.
\end{abstract}

Keywords: Traffic Grooming, WDM Networks, ADM, Unidirectional Rings, Approximation, Designs, Partition of graphs.

${ }^{*}$ This work has been partially funded by the European project IST FET AEOLUS, COST 293 Graal and MarieCurie RTN ADONET, and has been done in the context of the CRC Corso with France Telecom. 


\section{Introduction}

Traffic grooming is the generic term for packing low rate signals into higher speed streams (see the surveys $[7,14,22,24,30,21])$. By using traffic grooming, one can bypass the electronics in the nodes for which there is no traffic sourced or destinated to it and therefore reduce the cost of the network. Typically, in a WDM (Wavelength Division Multiplexing) network, instead of having one SONET Add Drop Multiplexer (ADM) on every wavelength at every node, it may be possible to have ADMs only for the wavelength used at that node (the other wavelengths being optically routed without electronic switching).

In SONET/WDM networks, we assign to each request $\{i, j\}$ a fraction of the bandwidth offered by a wavelength along a path from node $i$ to node $j$. If a given wavelength can carry at most $\mathrm{C}$ requests, we can assign to each request at most $\frac{1}{C}$ of the bandwidth. $\mathrm{C}$ is known as the grooming ratio. In the particular case of unidirectional rings, the routing is unique. Furthermore, if the traffic is symmetric, it can be easily shown (by exchanging wavelengths) that there always exists an optimal solution in which the same wavelength is given to a pair of symmetric requests. Then, without loss of generality, we will assign to each pair of symmetric requests, called a circle, a fraction of the bandwidth in the whole ring. In both cases, we need one ADM at node $i$ and one at node $j$. Also, two requests with a common extremity and assigned to the same wavelength will share an ADM. For example, if requests $\{1,2\}$ and $\{2,3\}$ are assigned to two different wavelengths, then we need 4 ADMs, while if they are assigned to the same wavelength we will need only 3 ADMs.

The so called traffic grooming problem consists of minimizing the total number of ADMs to be used (in order to reduce the overall cost of the network). Here we study the problem for an unidirectional SONET ring with $N$ nodes, a grooming ratio $C$, and an all-to-all uniform unitary traffic. This problem has been modeled as a graph partition problem in both [6] and [18]. The set of requests is modeled by a graph $I$, where $I=K_{N}$ in the all-to-all case. To a wavelength $w$ is associated a subgraph $B_{w}$ in which each edge corresponds to a request and each node to an ADM. The grooming constraint, that a wavelength can carry at most $C$ requests, corresponds to the fact that the number of edges $\left|E\left(B_{w}\right)\right|$ of each subgraph $B_{w}$ is at most $C$. The objective is therefore to minimize the total number of vertices used in the subgraphs.

Problem 1 (Grooming on unidirectional Cycle [6])

Given a number of nodes $N$ and a grooming ratio $C$ find a partition of the edges of the undirected graph $I=K_{N}$ into subgraphs $B_{w}, w=1, \ldots, W$ with $\left|E\left(B_{w}\right)\right| \leq C$ such that $\sum_{1 \leq w \leq W}\left|V\left(B_{w}\right)\right|$ is minimum.(This minimum will be denoted $A(C, N)$ ).

The traffic grooming problem has recently been extensively studied on unidirectional WDM rings, primarily in the context of variable traffic requirements $[10,13,18,25,28]$, but the case of fixed traffic requirements has served as an important special case $[3,4,5,6,7,8,14,16,17,19,20$, $22,26,29]$. The problem has also been studied on the path [2].

With a general set of requests, $I \neq K_{N}$, the grooming problem has been proved NP-Complete in unidirectional ring with grooming factor $C \geq 1[10,23]$. Then a first approximation algorithm for computing the total number of ADMs with approximation factor $\frac{C\left(1+\frac{1}{\Gamma C / 2\rceil}\right)}{\left\lceil\frac{1+\sqrt{1+8 C}}{2}\right\rceil}$, i.e. $\sim \sqrt{C}$, has been given in [18], and in [15] a $\log (C)$-approximation algorithm has been obtained. More recently, the grooming problem has been proved APX-Hard in [1] (i.e. there exists a constant $c$, such that Problem 1 can not be approximate within a factor $c$ ). 


\begin{tabular}{|l|c|c|c|c|c|c|c|c|c|c|}
\hline$C$ & 8 & 9 & 12 & 15 & 16 & 28 & 32 & 48 & 64 & 192 \\
\hline$\rho_{\max }(C)$ & $\frac{8}{5}$ & $\frac{9}{5}$ & 2 & $\frac{5}{2}$ & $\frac{5}{2}$ & $\frac{7}{2}$ & $\frac{32}{9}$ & $\frac{9}{2}$ & $\frac{16}{3}$ & $\frac{19}{2}$ \\
\hline$\gamma$ for Cons. 11 & 1.6 & 1.2 & 1.33 & 1.67 & 1.25 & 1.4 & 1.42 & 1.5 & 1.33 & 1.46 \\
\hline$\gamma$ for Cons. 18 & 1.2 & 1.2 & 1.17 & 1.33 & 1.25 & 1.37 & 1.3 & 1.31 & 1.33 & 1.39 \\
\hline$\gamma$ for Cons. 21 & 1.6 & 1.8 & 1 & 1.25 & 1.25 & 1.17 & 1.19 & 1.13 & 1.33 & 1.19 \\
\hline
\end{tabular}

Table 1: Approximation factor of the different constructions.

With the all-to-all set of requests, $I=K_{N}$, the extremal problem of finding the exact value of $A(C, N)$ is open and there is not even a conjecture for the extremal constructions. Optimal constructions for given grooming ratio $C$ were obtained using tools of graph and design theory [7, 11, 12], in particular for grooming ratio $C=3[3], C=4[19,8], C=5[5], C=6[4]$ and $C \geq N(N-1) / 6[8]$. However it will be a very long and intractable task to find optimal constructions for all grooming ratio. Existing heuristic algorithms [17, 26, 29] as well as the approximation algorithm proposed in $[15,18]$ are not satisfactory for the all-to-all case. Therefore, it is important to show that in this case we have approximation algorithms with a small approximation ratio.

In this paper, we will first present an asymptotical $1+\frac{4 C}{N}+o\left(\frac{1}{N}\right)$-approximation algorithm; unfortunately the construction is valid only for large $N$. Then we present a very simple construction using bipartite graphs which provides a $\gamma(C, N)$-approximation for the total number of ADMs, where $\gamma(C, N)$ is at most $\sqrt{2} \frac{\sqrt{C}}{[\sqrt{C}]}$ and in many cases better (for example, for $C=16: \gamma(16, N)=\frac{5}{4}$, and for $\left.C=64: \gamma(64, N)=\frac{4}{3}\right)$. Then we show several improvements of this construction by using other bipartite graphs or tripartite graphs (in that case $\gamma(C, N)$ is of order $\sqrt{\frac{3}{2}}$ ) or multipartite graphs. Values of the approximation factor obtained with different constructions are given in Table 1 for realistic values of $C$.

\section{Lower bound}

A tight lower bound for Problem 1 has been given in $[6,8]$ and is recalled in Theorem 2. The idea consists in using in the partition subgraphs which, for a given number of edges (less than $C)$, have the minimum number of vertices. So let us denote by $\rho(G)$ the ratio of a subgraph $G$, $\rho(G)=\frac{|E(G)|}{|V(G)|}$, and by $\rho(m)$ the maximum ratio of a subgraph with $m$ edges. Let finally $\rho_{\max }(C)$ denote the maximum possible ratio among all the subgraphs with $m \leq C$ edges, that is:

$$
\rho_{\max }(C)=\max \{\rho(G)|| E(G) \mid \leq C\}=\max _{m \leq C} \rho(m)
$$

Recall that $A(C, N)$ is the minimum number of ADM's needed in an unidirectional ring with the all-to-all set of request $\left(I=K_{N}\right)$ and with a grooming ratio $C . A(C, N)=\sum_{1 \leq w \leq W}\left|V\left(B_{w}\right)\right|$; 
so using $\rho_{\max }(C)\left|V\left(B_{w}\right)\right| \geq\left|E\left(B_{w}\right)\right|$ and $\sum_{1 \leq w \leq W}\left|E\left(B_{w}\right)\right|=\frac{N(N-1)}{2}$ we get the following lower bound:

Theorem 2 (Lower Bound [6]) $A(C, N) \geq \frac{N(N-1)}{2 \rho_{\max }(C)}$.

The value of $\rho_{\max }(C)$ has been evaluated in [6] and is recalled in Proposition 3.

\section{Proposition 3 ([6])}

- If $\frac{x(x-1)}{2} \leq C \leq \frac{(x+1)(x-1)}{2}$, then $\rho_{\max }(C)=\frac{x-1}{2}$ and the value is attained for $K_{x}$.

- If $\frac{(x+1)(x-1)}{2}<C<\frac{(x+1) x}{2}$, then $\rho_{\max }(C)=\frac{C}{x+1}$ and the value is attained for any graph with $C$ edges and $x+1$ vertices.

Values of $\rho_{\max }(C)$ are given in Table 1 for realistic values of $C$. The following corollary gives also a lower bound easier to manipulate.

Corollary $4 \rho_{\max }(C) \leq \sqrt{\frac{C}{2}}$ and so $A(C, N) \geq \frac{N(N-1)}{\sqrt{2 C}}$.

Proof : From Proposition 3, we know that $\rho_{\max }(C)=\frac{x-1}{2}$ (case 1$)$ or $\frac{C}{x+1}$ (case 2), and we can observe that $x=\left\lfloor\frac{1+\sqrt{1+8 C}}{2}\right\rfloor$. Thus we have

- case $1: 2 \rho_{\max }(C)=\left\lfloor\frac{1+\sqrt{1+8 C}}{2}\right\rfloor-1 \leq\left\lfloor\frac{\sqrt{1+8 C}-1}{2}\right\rfloor \leq \frac{\sqrt{8 C}}{2} \leq \sqrt{2 C}$ and so $\rho_{\max }(C) \leq \sqrt{\frac{C}{2}}$

- case $2: \rho_{\max }(C)=\frac{C}{\left[\frac{1+\sqrt{1+8 C}}{2}\right]+1} \leq \frac{C}{\frac{1+\sqrt{1+8 C}}{2}} \leq \sqrt{\frac{C}{2}}$

So $A(C, N) \geq \frac{N(N-1)}{\sqrt{2 C}}$.

\section{$3 \quad$ Asymptotic construction}

It has been shown in [6] that design theory can help to solve the grooming problem. In particular, a $G$-design of order $N$ (see [11] VI.24 or [9]) is nothing else than a partition of the edges of $K_{N}$ into subgraphs isomorphic to a given graph $G$. The interest of the existence of a $G$-design is shown by the following immediate proposition.

Proposition 5 ([6]) If there exists a $G$-design of order $N$, where $G$ is a graph with at most $C$ edges and with ratio $\rho_{\max }(C)$, then $A(C, N)=\frac{N(N-1)}{2 \rho_{\max }(C)}$.

Necessary conditions 6 (Existence of a $G$-design) If there exists a $G$-design, then

(i) $\frac{N(N-1)}{2}$ should be a multiple of $E(G)$

(ii) $N-1$ should be a multiple of the greatest common divisor of the degrees of the vertices of $G$. 
It has been shown that these conditions are sufficient for $C=3,6,10$ : $G$ being the complete graph $K_{3}, K_{4}$, or $K_{5}$ (in that case we have a "classical design", see [11] chapter II.3), and also for $C=15(N \geq 802): G$ being $K_{6}$. They are also sufficient for $C=4,5,8(N \neq 48), 9(N \geq 235)$ (see [11] chapter VI.24). More generally, Wilson [27] has shown that these necessary conditions are sufficient for any $C$ when $N$ is large enough. So we have the following Theorem:

Theorem 7 (see [6]) We have

- $A(3, N)=\frac{N(N-1)}{2}$ when $N \equiv 1$ or $3 \bmod 6$

- $A(4, N)=\frac{N(N-1)}{2}$ when $N \equiv 0$ or $1 \bmod 8$

- $A(5, N)=\frac{2 N(N-1)}{5}$ when $N \equiv 0$ or $1 \bmod 5$

- $A(6, N)=A(7, N)=\frac{N(N-1)}{3}$ when $N \equiv 1$ or $4 \bmod 12$

- $A(8, N)=\frac{5 N(N-1)}{16}$ when $N \equiv 0$ or $1 \bmod 16$, and $N \neq 48$

- $A(9, N)=\frac{5 N(N-1)}{18}$ when $N \equiv 0$ or $1 \bmod 9$, and $N \geq 235$

- $A(10, N)=\frac{N(N-1)}{4}$ when $N \equiv 1$ or $5 \bmod 20$

- $A(15, N)=A(16, N)=\frac{N(N-1)}{5}$ when $N \equiv 1$ or $6 \bmod 15$, and $N \geq 802$

Construction 8 For a given $C$, let $N_{2} \geq N$ be the smallest integer such that there exists a $G$ design where $G$ has at most $C$ edges and a ratio $\rho_{\max }(C)$. We obtain a valid construction for $N$ by removing $N_{2}-N$ nodes and the corresponding edges from the optimal construction for $N_{2}$.

In order to get an approximation factor of this solution, we need to know a lower bound for $A(C, N)$. A trivial lower bound is given by $A\left(C, N_{1}\right)$, where $N_{1} \leq N$ is the biggest integer such that there exists a $G$-design.

The following lemma allows to find values of $N_{1}$ and $N_{2}$ that are near to each other.

Lemma 9 Let $\alpha(C)$ be defined as follows:

- If $\frac{x(x-1)}{2} \leq C \leq \frac{(x+1)(x-1)}{2}$, then $\alpha(C)=x(x-1)$.

- If $\frac{(x+1)(x-1)}{2} \leq C<\frac{(x+1) x}{2}$, then $\alpha(C)=2 C$

Let $N_{1}=\alpha(C) t+1$ and $N_{2}=\alpha(C)(t+1)+1$ be such that $N_{1} \leq N \leq N_{2}$.

There always exists a graph $G$ with at most $C$ edges and ratio $\rho_{\max }(C)$ which satisfies Conditions 6 for $N_{1}$ and $N_{2}$.

Proof : When $\frac{x(x-1)}{2} \leq C \leq \frac{(x+1)(x-1)}{2}$, then $\rho_{\max }(C)$ is attained for $K_{x}$, and so let $G=K_{x}$. Both $N_{1}-1$ and $N_{2}-1$ are multiple of $\alpha(C)=x(x-1)$; and so the number of edges of $K_{N_{1}}$ (resp. $\left.K_{N_{2}}\right) \frac{N_{1}\left(N_{1}-1\right)}{2}$ (resp. $\frac{N_{2}\left(N_{2}-1\right)}{2}$ ) is a multiple of $E(G)=\frac{x(x-1)}{2}$. Condition (ii) is also satisfied as the degree of a vertex of $K_{N_{1}}$ (resp. $K_{N_{2}}$ ) $N_{1}-1$ (resp. $N_{2}-1$ ) is a multiple of $x-1$ the degree of $K_{x}$. 
When $\frac{(x+1)(x-1)}{2} \leq C<\frac{(x+1) x}{2}$, then $\rho_{\max }(C)$ is attained for any graph with $C$ edges and $x+1$ vertices. Let $r=\frac{(x+1) x}{2}-C$. So $0<r<x$. Let $G$ be the graph obtained from $K_{x+1}$ by removing the edges of a path of length $r$. $G$ has $C$ edges and so Condition (i) is satisfied as $\frac{N_{1}\left(N_{1}-1\right)}{2}=(2 C t+1) C t$ and $\frac{N_{2}\left(N_{2}-1\right)}{2}=(2 C(t+1)+1) C(t+1)$ are multiples of $E(G)$. As $0<r \leq x-1, G$ has a vertex which is not in the path that have been removed; this vertex has degree $x$, and the extremities of the path have degree $x-1$, so the greatest common divisor of the degrees of the vertices of $G$ is 1 . Condition (ii) is trivially satisfied.

Proposition 10 When $N$ is large enough to satisfy Wilson's Theorem, Construction 8 has an approximation factor $\gamma(C, N) \leq 1+\frac{4 C}{N}+o\left(\frac{1}{N}\right)$.

Proof: Let $f(C, N)$ denotes the number of ADMs obtained by Construction 8 and let $\gamma(C, N)=$ $\frac{f(C, N)}{A(C, N)}$ be its approximation factor. Let also $N_{1}$ and $N_{2}$, with $N_{1} \leq N \leq N_{2}$, be given by Lemma 9 . We have $A\left(C, N_{1}\right) \leq A(C, N) \leq f(C, N) \leq A\left(C, N_{2}\right)$ and $\gamma(C, N)=\frac{f(C, N)}{A(C, N)} \leq \frac{A\left(C, N_{2}\right)}{A\left(C, N_{1}\right)}=\frac{N_{2}\left(N_{2}-1\right)}{N_{1}\left(N_{1}-1\right)}$.

Since $N_{2}=\alpha(C)(t+1)+1=N_{1}+\alpha(C)$, we have $\gamma(C, N) \leq 1+\frac{2 \alpha(C)}{N_{1}}+\frac{\alpha(C)(\alpha(C)+1)}{N_{1}\left(N_{1}-1\right)}$.

Finally, as in both cases $\alpha(C) \leq 2 C$ and $N-N_{1} \leq \alpha(C)$, we obtain $\gamma(C, N) \leq 1+\frac{4 C}{N}+o\left(\frac{1}{N}\right)$.

Unfortunately, except for the small values of $C$ given in Theorem 7, the values of $N$ for which Wilson's Theorem and so Proposition 10 applies are very large. Furthermore, it is not known how to implement Construction 8 in polynomial time. So there is a need to find simpler and general constructions.

\section{Construction using bipartite graphs}

In this section, we first present a simple construction which gives an upper bound on the number of ADM's and we analyze it's approximation factor. Then, we present some improvements of this construction.

Basically our construction consists of partitioning the edges of $K_{N}$ into a maximum number of bipartite graphs, with at most $C$ edges, plus some small complete graphs. A complete-bipartite graph with 2 sets of $p$ nodes each has $p^{2}$ edges and a ratio of $\frac{p}{2}$. Therefore choosing $p^{2}$ to be $C$ or almost $C$ we get a ratio near to $\frac{\sqrt{C}}{2}$. As we will see in the proof of Proposition 12, the number of ADMs due to bipartite graphs dominates the total cost of the construction, and so the number of ADMs will be around $\frac{N(N-1)}{\sqrt{C}}$. From Theorem 2 we know that the lower bound is larger than $\frac{N(N-1)}{2 \rho_{\max }(C)}$. So our construction gives an approximation factor close to $\frac{2 \rho_{\max }(C)}{\sqrt{C}} \leq \sqrt{2}$ by Corollary 4 .

Several constructions are possible. We first present a basic construction (Construction 11) and then some improvements (Constructions 14 and 16) in order to have a precise approximation factor. We also give a variant in which $C$ is the product of two numbers.

\subsection{Basic construction}

Construction 11 Let $C=p^{2}+p^{\prime}, 0 \leq p^{\prime} \leq 2 p(p=\lfloor\sqrt{C}\rfloor) ;$ let $N=q p+r, 0 \leq r<p$, and let the vertices of $K_{N}$ be $V=\cup_{i=1}^{q} V_{i} \cup V_{q+1}$ with $\left|V_{i}\right|=p$ and $\left|V_{q+1}\right|=r$. 
We partition the edges of $K_{N}$ into $\frac{q(q-1)}{2} K_{p, p}$ on $V_{i} \cup V_{j}, 1 \leq i<j \leq q$, plus $q K_{p, r}$ on $V_{i} \cup V_{q+1}, 1 \leq i \leq q$, plus $q K_{p}$ on $V_{i}$ and one $K_{r}$ on $V_{q+1}$.

Proposition 12 Construction 11 is valid and uses $(q+1) N$ ADMs.

Proof : First all the subgraphs of the decomposition have at most $p^{2} \leq C$ edges. Since a bipartite graph $K_{x, y}$ has $x+y$ vertices and a complete graph $K_{x}$ has $x$ vertices, the number of ADMs involved in the construction is: $2 p \frac{q(q-1)}{2}+(p+r) q+q p+r=(q+1)(q p+r)=(q+1) N$ ADMs.

Corollary 13 When $C=p^{2}+p^{\prime}, 0 \leq p^{\prime} \leq 2 p$, and $N=q p+r, 0 \leq r<p$, Construction 11 provides a

$\frac{2 \rho_{\max }(C)}{[\sqrt{C}\rfloor}+O\left(\frac{1}{N}\right)<\sqrt{2} \frac{\sqrt{C}}{\lfloor\sqrt{C}\rfloor}+O\left(\frac{1}{N}\right)$-approximation of the number of ADMs.

Proof: Let $\gamma(C, N)$ be the approximation factor that is the ratio between the upper bound construction and the lower bound for a given grooming factor $C$. We know from Theorem 2 that $A(C, N) \geq \frac{N(N-1)}{2 \rho_{\max }(C)}$. So

$$
\gamma(C, N)=(q+1) N \frac{2 \rho_{\max }(C)}{N(N-1)}=2 \rho_{\max }(C) \frac{q+1}{N-1}
$$

Since $C=p^{2}+p^{\prime}$, we have $p=\lfloor\sqrt{C}\rfloor$ and $q=\frac{N-r}{p}=\frac{N-r}{\lfloor\sqrt{C}\rfloor}$. Thus we obtain

$$
\gamma(C, N)=\frac{2 \rho_{\max }(C)}{\lfloor\sqrt{C}\rfloor}\left(1+\frac{\lfloor\sqrt{C}\rfloor-r+1}{N-1}\right)
$$

\subsection{Improvements}

The above construction is very simple and provides a better approximation factor than [15]. The values of the approximation factor for some values of $C$ are indicated in Table 1. A first improvement can be obtained by noting that some bipartite subgraphs of the decomposition have strictly less than $C$ edges and therefore we can add to them some edges of the $K_{p}$ 's and of the $K_{r}$. That is always the case for the $K_{p, r}$ as $p r<p^{2} \leq C$ and also for the $K_{p, p}$ when $C>p^{2}$. Doing so we can get rid of the $O\left(\frac{1}{N}\right)$ in Corollary 13.

Construction 14 Let $C=p^{2}$ and $N=q p+r, 0<r<p$.

The construction consists of partitioning the edges of $K_{N}$ into $\frac{q(q-1)}{2} K_{p, p}$ on $V_{i} \cup V_{j}, 1 \leq i<$ $j \leq q$, plus $q$ subgraphs on $V_{i} \cup V_{q+1}, 1 \leq i \leq q$ containing the pr edges of the $K_{p, r}$ between $V_{i}$ and $V_{q+1}$ plus the $\frac{p(p-1)}{2}$ of the $K_{p}$ on $V_{i}$ and some edges of the $K_{r}$ on $V_{q+1}$.

Proposition 15 Let $C=p^{2}$ and $N=q p+r, 0<r<p$. If $\frac{r(r-1)}{2} \leq q\left(C-p r-\frac{p(p-1)}{2}\right)$, Construction 14 is valid and provides a $\frac{2 \rho_{\max }(C)}{\sqrt{C}} \leq \sqrt{2}$-approximation of the total number of ADMs. 
Proof : The subgraphs $K_{p, p}$ have $p^{2}=C$ edges. Each other subgraph contains the $p r$ edges of the $K_{p, r}$ between $V_{i}$ and $V_{q+1}$ plus the $\frac{p(p-1)}{2}$ of the $K_{p}$ on $V_{i}$. So we can still use $C-p r-\frac{p(p-1)}{2}>0$ edges of the $K_{r}$ on $V_{q+1}$; and altogether we can use all the edges of $K_{r}$ as $\frac{r(r-1)}{2} \leq q\left(C-p r-\frac{p(p-1)}{2}\right)$.

Construction 14 uses $q(q-1) p+q(p+r)=q(q p+r)=q N$ ADMs. So it has an approximation factor

$\gamma(C, N)=q N \frac{2 \rho_{\max }(C)}{N(N-1)}=\frac{2 \rho_{\max }(C)}{p} \frac{N-r}{N-1}$. Since $C=p^{2}$, we have $\gamma(C, N)=\frac{2 \rho_{\max }(C)}{\sqrt{C}}\left(1-\frac{r-1}{N-1}\right)$, and since $0<r$, we obtain $\gamma(C, N) \leq \frac{2 \rho_{\max }(C)}{\sqrt{C}} \leq \sqrt{2}$.

This strategy allows us to win a small amount of ADMs (at most $N$ ). For example, when $C=16, p=4$, and $q=4$, conditions of Construction 14 are satisfied for $r=1$ and 2 , so $N=17$ and $N=18$. For $N=17$ (resp. $N=18$ ), Construction 11 uses $5 \times 17=85$ (resp. $5 \times 18=90)$ ADMs and Construction 14 uses 68 (resp. 72) ADMs, that is a saving of 17 (resp. 18) ADMs.

When $C=p^{2}+p^{\prime}$, it is possible to improve Construction 14 by adding some edges of $K_{r}$ and of the $K_{p}$ 's to the subgraphs containing the bipartite graphs, thus reducing the total number of ADMs. In some cases, the subgraphs based on $K_{p}$ or $K_{r}$ may be completely absorbed as explained in the Construction 16 and Proposition 17.

Construction 16 Let $C=p^{2}+p^{\prime}, 0<p^{\prime} \leq 2 p$ and $N=q p+r, 0 \leq r<p$.

The construction consists of partitioning the edges of $K_{N}$ into $\frac{q(q-1)}{2}$ subgraphs on $V_{i} \cup V_{j}, 1 \leq$ $i<j \leq q$ containing the $p^{2}$ edges of the $K_{p, p}$ between $V_{i}$ and $V_{j}$ plus some edges of one of the $K_{p}$, plus $q$ subgraphs on $V_{i} \cup V_{q+1}, 1 \leq i \leq q$ containing the $p r$ edges of the $K_{p, r}$ between $V_{i}$ and $V_{q+1}$ plus some edges of the $K_{r}$ on $V_{q+1}$.

Proposition 17 Let $C=p^{2}+p^{\prime}, 0<p^{\prime} \leq 2 p$ and $N=q p+r, 0 \leq r<p$. If $(q-1) p^{\prime} \geq p(p-1)$, then Construction 16 is valid and provides a $\frac{2 \rho_{\max }(C)}{\lfloor\sqrt{C}\rfloor} \leq \sqrt{2} \frac{\sqrt{C}}{\lfloor\sqrt{C}\rfloor}$-approximation of the total number of ADMs.

Proof : The subgraphs on $V_{i} \cup V_{j}, 1 \leq i<j \leq q$ use the $p^{2}$ edges of $K_{p, p}$ and $p^{\prime}$ edges of one of the $K_{p}$. Altogether we can use all the edges of the $K_{p}$ as by the condition $\frac{q(q-1)}{2} p^{\prime} \geq \frac{p(p-1)}{2} q$. In each $K_{p, r}$ we can use $p^{2}+p^{\prime}-p r=p(p-r)+p^{\prime}$ edges of the $K_{r}$. Since we have $q K_{p, r}$ and that $q p^{\prime}>(q-1) p^{\prime}>p(p-1)>\frac{r(r-1)}{2}$, all edges of $K_{r}$ are used.

Construction 16 uses $q(q-1) p+q(p+r)=q(q p+r)=q N$ ADMs. So it has the desired approximation factor.

Note that condition of Proposition 17 is satisfied as soon as $q \geq p(p-1)$, that is $N \geq p^{2}(p-1)$.

Remark that in some cases the approximation factor can be strictly larger than $\sqrt{2}$ due to the integer part of $\sqrt{C}$. For example if $C=8,\lfloor\sqrt{C}\rfloor=2$ but $\rho_{\max }(C)=\frac{8}{5}$ and the approximation factor is $\frac{8}{5}=1.6>\sqrt{2}$. For $C=15,\lfloor\sqrt{C}\rfloor=3, \rho_{\max }(C)=\frac{5}{2}$ and the approximation factor is $\frac{5}{3}$.

\subsection{Case $C=p_{1} p_{2}$}

The next construction helps to deal with these cases where $C=p_{1} p_{2}$.

Construction 18 Let $C=p_{1} p_{2}+p^{\prime}, p_{1} \leq p_{2}$; let also $N=q p_{1} p_{2}+r, 0 \leq r<p_{1} p_{2}$. Let the vertices of $K_{N}$ be $V=\cup_{i=1}^{q} V_{i} \cup V_{q+1}$ with $\left|V_{i}\right|=p_{1} p_{2}$ and $\left|V_{q+1}\right|=r$. 
We partition the edges of $K_{N}$ into $\frac{q(q-1)}{2} K_{p_{1} p_{2}, p_{1} p_{2}}$ on $V_{i} \cup V_{j}, 1 \leq i<j \leq q$, plus $q K_{p_{1} p_{2}, r}$ on $V_{i} \cup V_{q+1}, 1 \leq i \leq q$, plus $q K_{p_{1} p_{2}}$ on $V_{i}$ and one $K_{r}$ on $V_{q+1}$.

Then we partition each $K_{p_{1} p_{2}, p_{1} p_{2}}$ into $p_{1} p_{2} K_{p_{1}, p_{2}}$ and each $K_{p_{1} p_{2}, r}$, where $r=\alpha_{1} p_{1}+\beta_{1}$, $0 \leq \beta_{1}<p_{1}$, into $p_{1} \alpha_{1} K_{p_{1}, p_{2}}$ plus $p_{1} K_{\beta_{1}, p_{2}}$.

Finally, we partition each $K_{p_{1} p_{2}}$ into $\frac{p_{2}\left(p_{2}-1\right)}{2} K_{p_{1}, p_{1}}$ plus $p_{2} K_{p_{1}}$, and each $K_{r}$ into $\frac{\alpha_{1}\left(\alpha_{1}-1\right)}{2}$ $K_{p_{1}, p_{1}}$ plus $\alpha_{1} K_{p_{1}, \beta_{1}}$ and $\alpha_{1} K_{p_{1}}$ and $1 K_{\beta_{1}}$.

Proposition 19 Let $C=p_{1} p_{2}+p^{\prime}$, and $N=q p_{1} p_{2}+r, 0 \leq r<p_{1} p_{2}$, Construction 18 is valid and provides a $\frac{\rho_{\max }(C)\left(p_{1}+p_{2}\right)}{p_{1} p_{2}}+O\left(\frac{1}{N}\right)$-approximation of the total number of ADMs.

Proof: As $\beta_{1}<p_{1}<p_{2}$, all the subgraphs of the decomposition, i.e. $K_{p_{1}, p_{2}}, K_{\beta_{1}, p_{2}}, K_{p_{1}, p_{1}}, K_{p_{1}}$, $K_{p_{1}, \beta_{1}}, K_{\beta_{1}}$, have at most $p_{1} p_{2} \leq C$ edges, and so the construction is valid.

The total number of ADMs is $\frac{q(q-1)}{2} p_{1} p_{2}\left(p_{1}+p_{2}\right)+q\left(\alpha_{1} p_{1}\left(p_{1}+p_{2}\right)+p_{1}\left(\beta_{1}+p_{2}\right)\right)+q p_{1} p_{2}^{2}+$ $\alpha_{1}\left(\alpha_{1}-1\right) p_{1}+\alpha_{1}\left(2 p_{1}+\beta_{1}\right)+\beta_{1}$. Using $N=q p_{1} p_{2}+r$ we get $\frac{N(N-1)\left(p_{1}+p_{2}\right)}{2 p_{1} p_{2}}+O(N)$ ADMs and so the approximation factor.

Remark : We can also modify the construction like we did before to include the edges of the $K_{p_{1} p_{2}}$ or $K_{r}$ in the bipartite subgraphs, and therefore get rid in many cases of the $O\left(\frac{1}{N}\right)$ in the approximation factor.

Note that when $p_{1}=p_{2}$, Construction 18 is exactly Construction 11 . However, we have more possible choices for $p_{1}, p_{2}$, and $p^{\prime}$, and so for many values Construction 18 is better than Construction 11. Of course we have interest to choose $p^{\prime}$ as small as possible, but also to choose $p_{1}$ and $p_{2}$ in order to minimize $\frac{p_{1}+p_{2}}{p_{1} p_{2}}$; that can be achieved by choosing $p_{1}$ and $p_{2}$ near to each other but not necessarily equals.

For example, let $C=32$. We can write $32=5 \times 5+7$, or $32=4 \times 8$, or $32=5 \times 6+2$. For $C=5 \times 5+7$, Construction 18 or 11 give approximation factor $\frac{2}{5} \rho_{\max }(C)$; if we choose $32=4 \times 8$ in Construction 18 we get an approximation factor $\frac{12}{32} \rho_{\max }(C)$ which is better since $\frac{12}{32}<\frac{2}{5}$. But we can do better using $32=5 \times 6+2$ in Construction 18 getting approximation factor $\frac{11}{30} \rho_{\max }(C)$.

Let now $C=8=2 \times 4, \rho_{\max }(C)=\frac{8}{5}$. With Construction 18 we get approximation factor is $\frac{8}{5} \frac{6}{8}=1.2$ to be compared with $\frac{8}{5}=1.6$ from Construction 11 . Similarly, when $C=15=3 \times 5$, $\rho_{\max }(C)=\frac{5}{2}$ and Construction 18 gives approximation factor $\frac{5}{2} \times \frac{8}{15}=\frac{4}{3}$ to be compared with $\frac{5}{3}$ from Construction 11.

\section{Construction with multipartite graphs}

In the previous section we have shown that using a partition of $K_{N}$ into small bipartite graphs, it is possible to obtain a $\frac{2 \rho_{\max }(C)}{\lfloor\sqrt{C}\rfloor}+O\left(\frac{1}{N}\right)$-approximation of the total number of ADMs. We will now show that using small multipartite graphs it is possible to drastically improve the approximation factor.

\subsection{Construction with tripartite graphs}

We will first use the optimal decomposition of $K_{N}$ obtained in [3] for a grooming factor $C=3$, and reported here in Theorem 20, to obtain a $\frac{\rho_{\max }(C)}{\left\lfloor\sqrt{\frac{C}{3}}\right\rfloor}+O\left(\frac{1}{N}\right)$-approximation algorithm. 
Theorem 20 (Theorem 4 of [3]) Let $n \geq 2$. There exists a partition of $K_{n}$ using

- if $n \equiv 1,3 \bmod 6, \frac{n(n-1)}{6} K_{3}$

- if $n \equiv 5 \bmod 6, \frac{n(n-1)-8}{6} K_{3}$ and $2 P_{3}$

- if $n \equiv 0,4 \bmod 12, \frac{n(n-1)}{6}-\frac{n}{4} K_{3}$ and $\frac{n}{4} K_{1,3}$

- if $n \equiv 2 \bmod 6, \frac{n(n-1)-2}{6}-\left\lceil\frac{n-2}{4}\right\rceil K_{3},\left\lceil\frac{n-2}{4}\right\rceil K_{1,3}$ and 1 edge

- if $n \equiv 6,10 \bmod 12, \frac{n(n-1)}{6}-\frac{n+2}{4} K_{3}, \frac{n-2}{4} K_{1,3}$ and $1 P_{4}$

where $P_{3}$ is a path with 3 vertices, $P_{4}$ a path with 4 vertices and $K_{1,3}$ a complete bipartite graph between a set of size 1 and a set of size 3 (also call a claw or a 3-star).

Construction 21 Let $C=3 p^{2}+p^{\prime}, 0 \leq p^{\prime}<6 p+3$ and $N=q p+r, 1 \leq r \leq p$, and let the vertices of $K_{N}$ be $\cup_{i=1}^{q} V_{i} \cup V_{q+1}$, with $\left|V_{i}\right|=p$ and $\left|V_{q+1}\right|=r$.

Consider $K_{q+1}$; replace each node $i$ of $K_{q+1}$ by the set of nodes $V_{i}, 1 \leq i \leq q+1$, and each edge $\{i, j\}$ by the corresponding $K_{p, p}$ or $K_{p, r}$ constructed on $V_{i} \cup V_{j}$. Now consider the partition of $K_{q+1}$ given by Theorem 20. To each subgraph of the partition we associate in $K_{N}$ a multipartite subgraph of $K_{N}$; for example to a $K_{3}(i, j, k)$ will correspond a tripartite $K_{p, p, p}$ built on $V_{i} \cup V_{j} \cup V_{k}$. All these subgraphs plus the $q K_{p}$ built on $V_{i}, 1 \leq i \leq q$ and the $K_{r}$ built on $V_{q+1}$ form a partition of $K_{N}$.

Proposition 22 Construction 21 is valid and provides a $\frac{\rho_{\max }(C)}{\left[\sqrt{\frac{C}{3}}\right]}+O\left(\frac{1}{N}\right)$-approximation of the total number of ADMs.

Proof: All the subgraphs of the partition of $K_{q+1}$ contains at most 3 edges and so the corresponding subgraphs in $K_{N}$ have at most $3 p^{2} \leq C$ edges. The $K_{p}$ and $K_{r}$ have at most $\frac{p(p-1)}{2}<C$ edges. So Construction 21 is valid.

We can count exactly the number of ADMs.

- When $q+1 \equiv 1,3 \bmod 6$, each node of $K_{q+1}$ appears in $\frac{q}{2} K_{3}$. So each node of $K_{N}$ appears in $\frac{q}{2}+1$ subgraphs (the +1 coming from the $K_{p}$ or $K_{r}$ to which it belongs) and so we have $\frac{q+2}{2} N$ ADMs.

- When $q+1 \equiv 5 \bmod 6$ and according to the proof of Theorem 20 of [3], the two $P_{3}$ of the partition of $K_{q+1}$ contain the edges $x-u, u-y$ and $x-v, v-y$. Nodes different from $x$ and $y$ appear in $\frac{q}{2}$ subgraphs of the partition and node $x$ and $y$ in $\frac{q}{2}+1$ subgraphs. As nodes $x$ and $y$ are replaced by at most $p$ vertices, all nodes of $K_{N}$ appear in $\frac{q}{2}$ subgraphs except at most $2 p$ of them which appear in one more. So altogether we have at most $\frac{q+2}{2} N+2 p$ ADMs.

- When $q+1 \equiv 0,4 \bmod 12, \frac{q+1}{4}$ nodes of $K_{q+1}$ appears in $\frac{q-3}{2} K_{3}$ and one $K_{1,3}$ (namely the central vertices of the $\left.K_{1,3}\right)$ and $\frac{3(q+1)}{4}$ appear in $\frac{q-1}{2} K_{3}$ and one $K_{1,3}$. As the nodes are replaced by at most $p$ vertices, each node of $K_{N}$ appear in $\frac{q+1}{2}$ subgraphs and at most $\frac{3 p(q+1)}{4}$ in one more subgraph. So altogether we have $\frac{q+1}{2} N+p \frac{3 p(q+1)}{4}$ ADMs. 
- When $q+1 \equiv 2 \bmod 6$, a similar analysis gives that we have at most $\frac{q+1}{2} N+3 p\left\lceil\frac{q-1}{4}\right\rceil+2 p$ ADMs.

- When $q+1 \equiv 6,10 \bmod 12$, we have at $\operatorname{most} \frac{q+1}{2} N+3 p \frac{q-1}{4}+2 p$ ADMs.

In all the cases the total number of $\mathrm{ADMs}$ is $\frac{N(N-1)}{2 p}+O(N)$ giving the proposition as $p=\left\lfloor\sqrt{\frac{C}{3}}\right\rfloor$

Note that we can in some cases get rid of the $O\left(\frac{1}{N}\right)$ like we did in Constructions 14 or 16 and in particular if $C=3 p^{2}+p^{\prime}, 0<p^{\prime}<6 p+3$ for all values of $N$ large enough.

\subsection{Alternative constructions with tripartite graphs}

We could have also used instead of the partition of $K_{q+1}$ of Theorem 20, a covering of the edges of $K_{q+1}$ with $K_{3}$ 's. Indeed it is known that the edges of $K_{n}$ can be covered by $\left\lceil\frac{n}{3}\left\lceil\frac{n-1}{2}\right\rceil\right\rceil K_{3}$ 's (see [11] chapter VI.11). So we obtain the following construction.

Construction 23 Let $C=3 p^{2}+p^{\prime}, 0 \leq p^{\prime}<6 p+3$ and $N=q p+r, 1 \leq r \leq p$, and let the vertices of $K_{N}$ be $\cup_{i=1}^{q} V_{i} \cup V_{q+1}$, with $\left|V_{i}\right|=p$ and $\left|V_{q+1}\right|=r$.

Replace each vertex of $K_{q+1}$ by the set of nodes $V_{i}, 1 \leq i \leq q+1$ and each edge by the corresponding $K_{p, p}$ or $K_{p, r}$. From a covering of the edges of $K_{q+1}$ with $K_{3}$ 's we obtain a covering of the edges of $K_{N}$ with $K_{p, p, p}$ or $K_{p, p, r}$ plus the $K_{p}$ on the $V_{i}, 1 \leq i \leq q$, and the $K_{r}$ on $V_{q+1}$.

Proposition 24 Construction 23 is valid and gives a $\frac{\rho_{\max }(C)}{\left[\sqrt{\frac{C}{3}}\right]}+O\left(\frac{1}{N}\right)$-approximation of the number of ADMs.

Proof: The subgraphs in the covering have at most $3 p^{2}$ edges and so the construction is valid. Each node of $K_{N}$ belongs to at most $\left\lceil\frac{q}{2}\right\rceil+1$ subgraphs and so the partition of $K_{N}$ uses $\frac{N(N-1)}{2 p}+O(N)$ ADMs.

In some cases we can also use other partitions based on partitions in tripartite graphs.

Construction 25 Let $C=3 p^{2}$ and $N=3^{a} p, a \geq 1$.

From the existence of 3-GDD of type $u^{3}$ (see [11] chapter IV.4), that is a partition of the tripartite graph $K_{u, u, u}$ into $K_{3}, u \geq 1$, we know that $K_{u p, u p, u p}$ can be partition into $u^{2} K_{p, p, p}$. Thus, we partition the edges of $K_{N}$ as follows

1. If $N=3 p$ (i.e. $a=1$ ), partition $K_{3 p}$ into one $K_{p, p, p}$ and $3 K_{p}$.

2. Otherwise

(a) Partition the edges of $K_{N}$ into $3 K_{3^{a-1} p}$ and one $K_{3^{a-1} p, 3^{a-1} p, 3^{a-1} p}$

(b) Partition $K_{3^{a-1} p, 3^{a-1} p, 3^{a-1} p}$ into $\left(3^{a-1}\right)^{2} K_{p, p, p}$

(c) Repeat the process on each $K_{3^{a-1} p}$

One can check that we have partitioned $K_{N}$ into $\sum_{i=0}^{a-1} 3^{i}\left(3^{a-i-1}\right)^{2}=\frac{3^{a}\left(3^{a}-1\right)}{6}=\frac{N(N-p)}{6 p^{2}} K_{p, p, p}$ and $3^{a}=\frac{N}{p} K_{p}$. 
Proposition 26 Construction 25 uses $\frac{N(N+p)}{2 p}$ ADMs and provide a $\frac{\rho_{\max }(C)}{\sqrt{\frac{C}{3}}}+O\left(\frac{1}{N}\right)$-approximation of the total number of ADMs.

Proof : Construction 25 uses $3 p \frac{N(N-p)}{6 p^{2}}+p \frac{N}{p}=\frac{N(N+p)}{2 p}$ ADMs. Thus it has approximation factor $\gamma(C, N)=\frac{N(N+p)}{p} \frac{\rho_{\max }(C)}{N(N-1)}=\frac{\rho_{\max }(C)}{\sqrt{\frac{C}{3}}}\left(1+\frac{p+1}{N-1}\right)$.

Remark that Construction 25 gives the same approximation ratio than Construction 21, but it can be better for some particular values of $C$ and $N$. For example, when $C=12$ and $N=18$ Construction 25 uses 90 ADMs, while Construction 21 uses 106 ADMs.

\subsection{Construction with multipartite graphs}

Finally, we can use partitions with 4 partite (resp. 5 partite) graphs using partitions or coverings of $K_{q+1}$ with $K_{4}$ (resp. $K_{5}$ ). For example it is known that the edges of $K_{q+1}$ can be covered with $\left\lceil\frac{q+1}{4}\left\lceil\frac{q}{3}\right\rceil\right\rceil K_{4}$ 's for $q+1>19$ or $\left\lceil\frac{q+1}{5}\left\lceil\frac{q}{4}\right\rceil\right\rceil K_{5}$ 's for $q+1>429$ (see [11] chapter VI.11.4). Replacing each vertex $i$ of $K_{q+1}$ by the set $V_{i}$ and the edges by the corresponding $K_{p, p}$ or $K_{p, r}$, we get respectively a covering of the edges of $K_{N}$ with 4-partite subgraphs $K_{p, p, p, p}$ or $K_{p, p, p, r}$ (or 5-partite subgraphs). We obtain respectively a total number of $\frac{N(N-1)}{3 p}+O(N)$ ADMs, or $\frac{N(N-1)}{4 p}+O(N)$ ADMs. We summarize the results in the next proposition.

Proposition 27 Let $C=6 p^{2}+p^{\prime}, 0 \leq p^{\prime}<12 p+6$, and $N=q p+r, 1 \leq r \leq p$. We have a $\frac{\rho_{\max }(C)}{\left\lfloor\sqrt{\frac{3 C}{8}}\right\rfloor}+O\left(\frac{1}{N}\right)$ approximation.

Let $C=10 p^{2}+p^{\prime}, 0 \leq p^{\prime}<20 p+10$, and $N=q p+r, 1 \leq r \leq p$. We have a $\frac{\rho_{\max }(C)}{\left[\sqrt{\frac{2 C}{5}}\right]}+O\left(\frac{1}{N}\right)$ approximation.

Using $\rho_{\max }(C) \leq \sqrt{\frac{C}{2}}$, we have a $\sqrt{\frac{4}{3}}+O\left(\frac{1}{N}\right)$-approximation or a $\sqrt{\frac{5}{4}}+O\left(\frac{1}{N}\right)$-approximation.

We can also give a construction analogous to Construction 21 using the optimal construction for a grooming factor $C=6$ presented in [4]. The results are a little better but give the same order of approximation.

We can also generalize Construction 25 when $C=6 p^{2}$ and $N=4^{a} p$, using a partition of $K_{u p, u p, u p, u p}$ into $u^{2} K_{p, p, p, p}$. We will obtain a $\sqrt{\frac{4}{3}}+O\left(\frac{1}{N}\right)$-approximation. Similarly, when $C=10 p^{2}$ and $N=5^{a} p$ we will obtain a $\sqrt{\frac{5}{4}}+O\left(\frac{1}{N}\right)$-approximation, and more generally, when $C=\frac{\alpha(\alpha-1)}{2} p^{2}$ and $N=\alpha^{a} p$ we will obtain a $\sqrt{\frac{\alpha}{\alpha-1}}+O\left(\frac{1}{N}\right)$-approximation. Unfortunately, such constructions apply only for a few values of $N$. Also, the constructions that we have presented in Section 4 and 5.1 or 5.2 are more interesting in practice.

\section{Comparison between constructions}

We have presented various constructions, but none of them is always better than the others. According to the values of $C$ and $N$, one has to choose the most efficient construction. To illustrate that, we have written a program that computes for any $C$ and $N$ the values of all constructions, 
and we have reported some results in Figure 1. The results of the program show that for small values of $C$ and $N$ there is no absolute winner. For given $C$ and large value of $N$, Figure 1 confirms the asymptotic results of Table 1. For example, when $C=16$, Figure 1(b) shows that all the constructions are equivalent with a slight advantage for Construction 14. However, we know from Theorem 7 that starting from $N=802$, Construction 8 will always be better. For $C=12,32$, Construction 21 is the best (except for spare values where Constructions 8 and 25 apply). For given $N$, Figure 1(d) shows again that there is no absolute winner and the importance of divisibility condition on $C$ like $C$ near to $p^{2}$ (see the isolated point of Construction 14 for $C=64$ ) or $3 p^{2}$ or $6 p^{2}$.

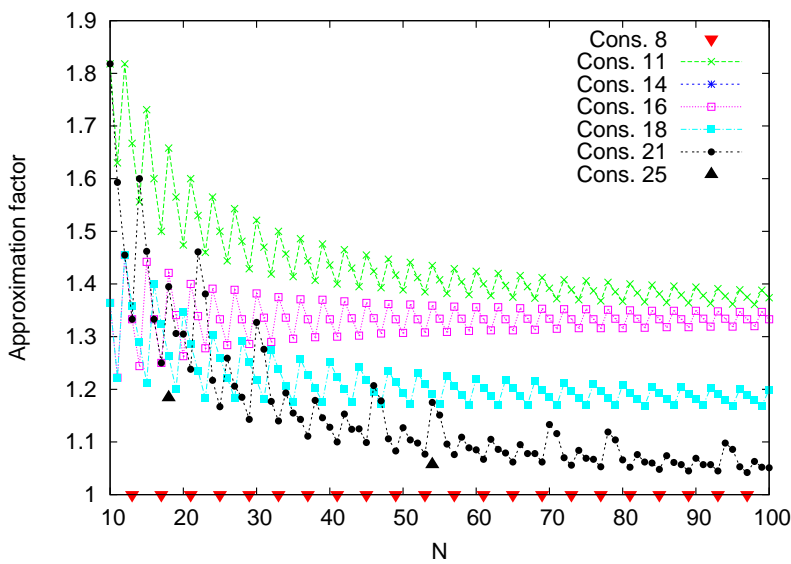

(a) $C=12$

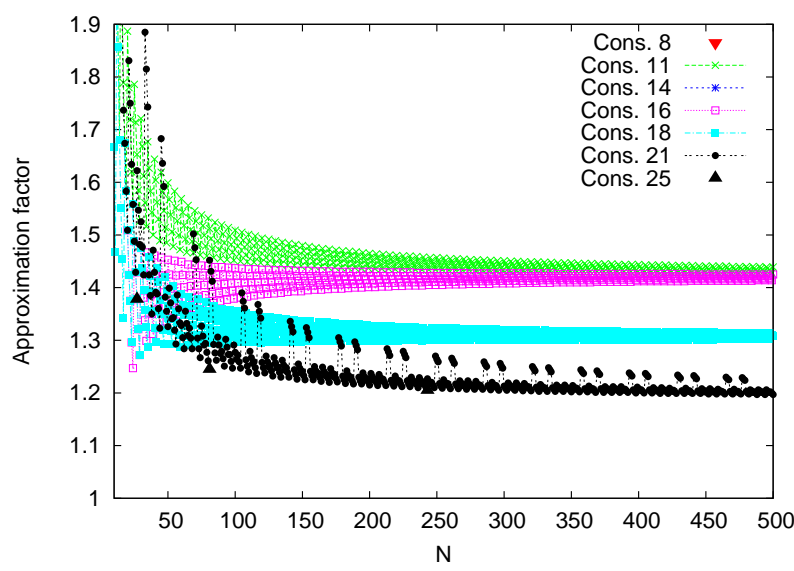

(c) $C=32$

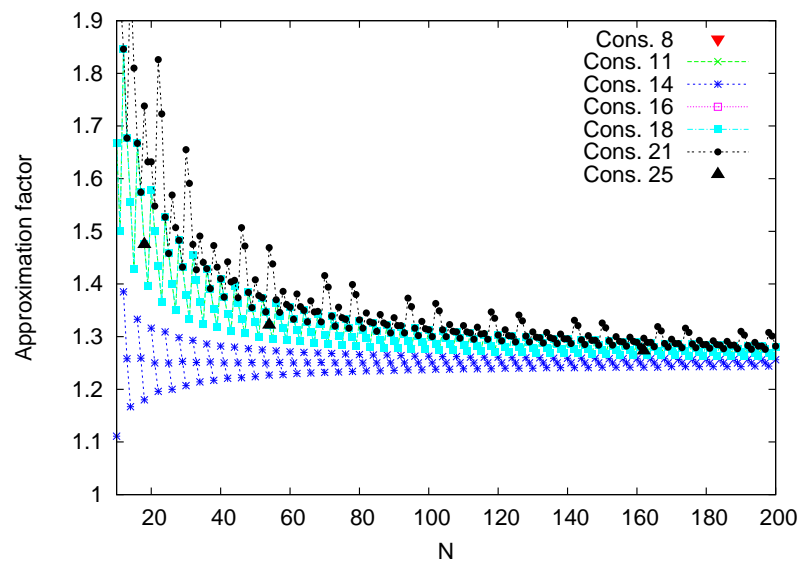

(b) $C=16$

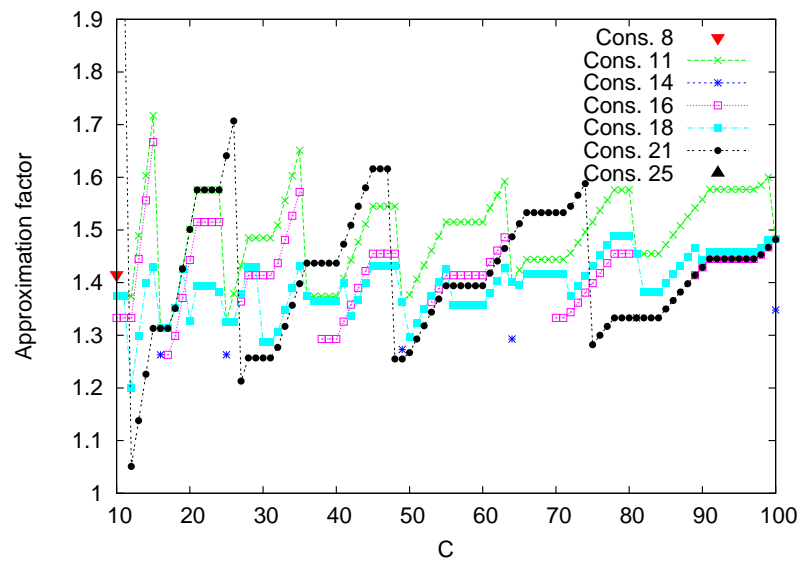

(d) $N=100$

Figure 1: Approximation ratio for given $C(\mathrm{a}, \mathrm{b}, \mathrm{c})$ or given $N(\mathrm{~d})$. 


\section{Conclusion}

In this paper, using tools of design theory, we have given different constructions with a small approximation factor for all-to-all traffic grooming in unidirectional ring. These simple constructions might also be used to compute good solutions for very dense set of requests, i.e. instances that are almost all-to-all, for which only $O(\log C)$-approximation algorithms are known so far. The traffic grooming problem being APX-Hard [1], this work represents an important step toward the conception of tight approximation algorithms for practical instances.

\section{Acknowledgments}

We would like to thank Charles J. Colbourn and Ignasi Sau Valls for fruitful discussions and the anonymous referees for very helpful comments.

\section{References}

[1] O. Amini, S. Pérennes, and I. Sau Valls, Hardness and approximation of traffic grooming, 18th International Symposium on Algorithms and Computation (ISAAC 2007) (Sendai, Japan), December 2007, pp. 561-573.

[2] J-C. Bermond, L. Braud, and D. Coudert, Traffic grooming on the path, Theoretical Computer Science 384 (2007), no. 2-3, 139-151.

[3] J-C. Bermond and S. Ceroi, Minimizing SONET ADMs in unidirectional WDM ring with grooming ratio 3, Networks 41 (2003), no. 2, 83-86.

[4] J-C. Bermond, C. Colbourn, D. Coudert, G. Ge, A. Ling, and X. Muñoz, Traffic grooming in unidirectional WDM rings with grooming ratio $C=6$, SIAM Journal on Discrete Mathematics 19 (2005), no. 2, 523-542.

[5] J.-C. Bermond, C.J. Colbourn, A.C.H. Ling, and M.-L. Yu, Grooming in unidirectional rings: $K_{4}-e$ designs, Discrete Mathematics, Lindner's Volume 284 (2004), no. 1-3, 57-62.

[6] J-C. Bermond and D. Coudert, Traffic grooming in unidirectional WDM ring networks using design theory, IEEE ICC (Anchorage, Alaska), vol. 2, May 2003, ON07-3, pp. 1402-1406.

[7] _ The CRC handbook of combinatorial designs (2nd edition), C.J. Colbourn and J.H. Dinitz ed., Discrete Mathematics and Its Applications, vol. 42, ch. VI.27, Grooming, pp. 493496, CRC Press, November 2006.

[8] J-C. Bermond, D. Coudert, and X. Muñoz, Traffic grooming in unidirectional WDM ring networks: the all-to-all unitary case, The 7th IFIP Working Conference on Optical Network Design \& Modelling - ONDM'03, February 2003, pp. 1135-1153.

[9] J-C. Bermond and D. Sotteau, Graph decompositions and G-designs, 5th British Combinatorial conference (Aberdeen), Congressus Numerantium 15 Utilitas math. Pub., 1975, pp. 53-72. 
[10] A. L. Chiu and E. H. Modiano, Traffic grooming algorithms for reducing electronic multiplexing costs in WDM ring networks, IEEE/OSA Journal of Lightwave Technology 18 (2000), no. 1, $2-12$.

[11] C.J. Colbourn and J.H. Dinitz (eds.), The CRC handbook of Combinatorial designs (2nd edition), vol. 42, CRC Press, November 2006.

[12] J. Dinitz and D. Stinson (eds.), Contemporary design theory: A collection of Surveys, Discrete Mathematics and Optimization, Wiley-Interscience, 1992.

[13] R. Dutta and N. Rouskas, On optimal traffic grooming in WDM rings, IEEE Journal of Selected Areas in Communications 20 (2002), no. 1, 1-12.

[14] _ Traffic grooming in WDM networks: Past and future, IEEE Network 16 (2002), no. 6, $46-56$.

[15] M. Flammini, L. Moscardelli M. Shalom, and S. Zaks, Approximating the traffic grooming problem, 16th Annual International Conference on Algortihms and Computation (ISAAC), Lecture Notes in Computer Science, vol. 3827, Springer Berlin / Heidelberg, December 2005, pp. 915-924.

[16] O. Gerstel, P. Lin, and G. Sasaki, Wavelength assignment in a WDM ring to minimize cost of embedded SONET rings, IEEE INFOCOM (San Francisco, California), vol. 1, March 1998, pp. 94-101.

[17] O. Gerstel, R. Ramaswani, and G. Sasaki, Cost-effective traffic grooming in WDM rings, IEEE/ACM Transactions on Networking 8 (2000), no. 5, 618-630.

[18] O. Goldschmidt, D. Hochbaum, A. Levin, and E. Olinick, The SONET edge-partition problem, Networks 41 (2003), no. 1, 13-23.

[19] J.Q. Hu, Optimal traffic grooming for wavelength-division-multiplexing rings with all-to-all uniform traffic, OSA Journal of Optical Networks 1 (2002), no. 1, 32-42.

[20] _ Traffic grooming in wdm ring networks: A linear programming solution, OSA Journal of Optical Networks 1 (2002), no. 11, 397-408.

[21] S. Huang, R. Dutta, and G. N. Rouskas, Traffic grooming in path, star, and tree networks: Complexity, bounds, and algorithms, IEEE Journal on Selected Areas in Communications 24 (2006), no. 4, 66-82.

[22] E. Modiano and P. Lin, Traffic grooming in WDM networks, IEEE Communications Magazine 39 (2001), no. 7, 124-129.

[23] M. Shalom, W. Unger, and S. Zaks, On the complexity of the traffic grooming problem in optical networks, 4th International Conference on Fun With Algorithms (Castiglioncello (LI), Tuscany, Italy), Lecture Notes in Computer Science, vol. 4475, Springer Berlin / Heidelberg, June 2007, pp. 262-271. 
[24] A. Somani, Survivable traffic grooming in WDM networks, Broad band optical fiber communications technology - BBOFCT (Jalgaon, India) (D.K. Gautam, ed.), Nirtali Prakashan, December 2001, Invited paper, pp. 17-45.

[25] P-J. Wan, G. Calinescu, L. Liu, and O. Frieder, Grooming of arbitrary traffic in SONET/WDM BLSRs, IEEE Journal of Selected Areas in Communications 18 (2000), no. 10, 1995-2003.

[26] J. Wang, W. Cho, V. Vemuri, and B. Mukherjee, Improved approches for cost-effective traffic grooming in WDM ring networks: Ilp formulations and single-hop and multihop connections, IEEE/OSA Journal of Lightwave Technology 19 (2001), no. 11, 1645-1653.

[27] R.M. Wilson, Decomposition of complete graphs into subgraphs isomorphic to a given graph, Congressus numerantium 15 (1976), 647-659.

[28] X. Yuan and A. Fulay, Wavelength assignment to minimize the number of SONET ADMs in WDM rings, Photonic Network Communications 5 (2003), no. 1, 59-68.

[29] X. Zhang and C. Qiao, An effective and comprehensive approach for traffic grooming and wavelength assignment in SONET/WDM rings, IEEE/ACM Transactions on Networking 8 (2000), no. 5, 608-617.

[30] K. Zhu and B. Mukherjee, A review of traffic grooming in WDM optical networks: Architectures and challenges, Optical Networks Magazine 4 (2003), no. 2, 55-64. 